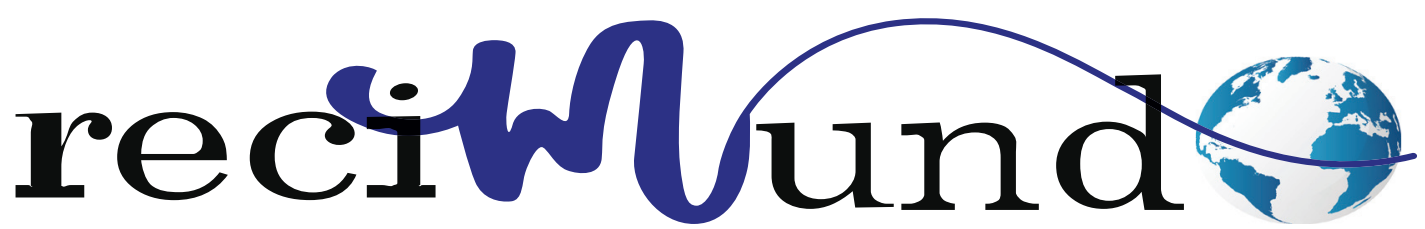

Revista Científica Mundo de la Investigación y el Conocimiento

DOI: 10.26820/recimundo/4.(4).octubre.2020.531-538

URL: http://recimundo.com/index.php/es/article/view/1007

EDITORIAL: Saberes del Conocimiento

REVISTA: RECIMUNDO

ISSN: 2588-073X

TIPO DE INVESTIGACióN: Artículo de Revisión

Código UNESCO: 32 Ciencias Médicas

PAGINAS: $531-538$

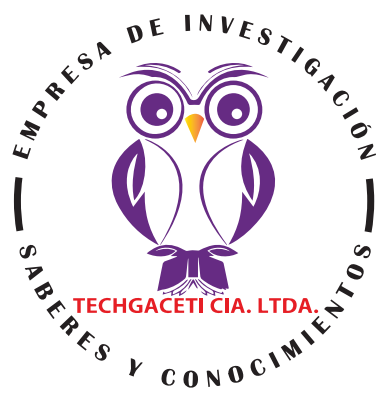

\title{
Cuidados quirúrgicos a pacientes sometidos a una mamoplastia
}

Surgical care for patients undergoing mammoplasty

Cuidados cirúrgicos para pacientes submetidos à mamoplastia

Fressia Carolina Zambrano Zambrano'; Marcos Antonio Campozano Burgos²; Félix Patricio Cedeño Flores;

Luis Gustavo Chávez Heredia ${ }^{4}$

RECIBIDO: 10/11/2020 ACEPTADO: 26/11/2020 PUBLICADO: 13/12/2020

1. Médico Cirujano de la Universidad Laica Eloy Alfaro de Manabí; Egresada en Master Universitario en Dirección y Gestión Sanitaria; Médico General de primer nivel de Atención Primaria; Centro de Salud Plan de Vivienda Sto. Domingo; Ecuador; fressia2312@gmail.com; https://orcid.org/0000-0003-4226-1156

2. Médico Cirujano de la Universidad Laica Eloy Alfaro de Manabí; IESS Portoviejo; Ecuador; drmarcocb.03@gmail.com; https://orcid.org/0000-0002-0216-1567

3. Médico Cirujano de la Universidad Laica Eloy Alfaro de Manabí; Hospital de Especialidades; Portoviejo; Ecuador; felix_1991cd@hotmail.es; https://orcid.org/0000-0003-3293-8223

4. Médico Cirujano de la Universidad Laica Eloy Alfaro de Manabí; Hospital IESS Manta; Ecuador; drgusy@gmail.com; https:// orcid.org/0000-0002-4257-9845

CORRESPONDENCIA

Fressia Carolina Zambrano Zambrano

fressia2312@gmail.com

Sto. Domingo; Ecuador

๑ RECIMUNDO; Editorial Saberes del Conocimiento, 2020 


\title{
RESUMEN
}

La mamoplastia es un procedimiento que puede ser tanto de aumento como de reducción de senos, en la mayoría de los casos se realiza es el primero, con lo que puede catalogarse como un procedimiento de ciruja plástica. Sin embargo, hay casos donde este procedimiento se realiza por daños ocasionados por implantes de mala calidad, rechazo del cuerpo, entre otros, que es donde se realiza la mamoplastia de reducción, pero también este ‘procedimiento se realiza luego de una mastectomía como consecuencia de un cáncer de mama. En líneas generales estos procedimientos tienen muy bajas tasas de incidencias, las cuales, de aparecer, se presentan luego la alta médica, hematomas, infecciones, son las más comunes. Los cuidados claves son los que el médico tratante le indica a la paciente que debe realizar en casa como dormir de lado para no ejercer presión sobre el seno, guardar reposo para evitar la rotación de la prótesis, así como la medicación indicada que generalmente es analgésicos, antinflamatorios y relajantes musculares. Los cuidados quirúrgicos están más asociados al momento de realizarse la operación cuando los instrumentos y todos los elementos necesarios que se necesitan deben ser manipulados con la debida asepsia y esterilización.

Palabras clave: Analgésicos, senos, prótesis, hematoma, infección.

\begin{abstract}
Mammoplasty is a procedure that can be both breast augmentation and reduction, in most cases it is performed first, which can be classified as a plastic surgery procedure. However, there are cases where this procedure is performed due to damage caused by poor quality implants, rejection of the body, among others, which is where reduction mammoplasty is performed, but this procedure is also performed after a mastectomy as a result of a breast cancer. In general, these procedures have very low incidence rates, which, if they appear, are then discharged, bruises, infections, are the most common. The key cares are those that the treating physician tells the patient to do at home, such as sleeping on her side to avoid putting pressure on the breast, resting to avoid the rotation of the prosthesis, as well as the indicated medication, which is generally analgesics. , anti-inflammatory and muscle relaxants. Surgical care is more associated with the time of the operation when the instruments and all the necessary elements that are needed must be handled with due asepsis and sterilization.
\end{abstract}

Keywords: Analgesics, breasts, prosthesis, hematoma, infection.

\section{RESUMO}

A mamoplastia é um procedimento que pode ser tanto de aumento como de redução dos seios, na maioria dos casos é realizada primeiro, o que pode ser classificado como um procedimento de cirurgia plástica. Entretanto, há casos em que este procedimento é realizado devido a danos causados por implantes de má qualidade, rejeição do corpo, entre outros, que é onde a mamoplastia de redução é realizada, mas este procedimento também é realizado após uma mastectomia como resultado de um câncer de mama. Em geral, estes procedimentos têm taxas de incidência muito baixas, que, se aparecerem, são então liberadas, hematomas, infecções, são os mais comuns. Os principais cuidados são aqueles que o médico tratante diz à paciente para fazer em casa, como dormir de lado para evitar pressionar a mama, descansar para evitar a rotação da prótese, assim como a medicação indicada, que é geralmente analgésica. A paciente deve usar anti-inflamatórios, anti-inflamatórios e relaxantes musculares. O cuidado cirúrgico está mais associado ao tempo da operação quando os instrumentos e todos os elementos necessários devem ser tratados com a devida assepsia e esterilização.

Palavras-chave: Analgésicos, seios, próteses, hematoma, infecção. 


\section{Introducción}

Conforme a datos de la Organización Mundial de la Salud (OMS), se estima que del 8 al 9 por ciento de las mujeres, desarrollarán a lo largo de su vida cáncer de mama, la principal causa de mortalidad en las mujeres. Es bien conocido el miedo que la palabra o el diagnóstico de cáncer genera en la población. Sin embargo, con el desarrollo científico y tecnológico cada vez se obtiene mayor supervivencia al mismo, pero presentando una serie de secuelas o complicaciones del propio tratamiento (Aguinaga Pinto, 2015).

La mamoplastia de aumento es uno de los procedimientos más populares y de mayor prevalencia en cirugía plástica estética. En Estados Unidos la ASPS3 describe que esta intervención ocupa el primer puesto de las cirugías estéticas con una frecuencia de 289.000 mujeres sometidas a mamoplastia de aumento durante el año 2009. Sin embargo es importante considerar que el número de estos procedimientos a nivel mundial es mucho mayor ya que la oferta del turismo medico ha incentivado con bajos costos a que las personas puedan acceder a la realización de cualquier cirugía estética en países que han ganado reconocimiento por su calidad en resultados a costos mucho más bajos que en los países desarrollados (Prieto, Jerez, Pineda, \& Robayo, 2014, pág. 38). La operación no garantiza un éxito total, pero si un avance, dentro de los cuidados post operatorios el paciente debe seguirlos con disciplina, alcanzar la mejoría esperada e incorporar una serie de cambios en el estilo de vida de la mujer y posiblemente a su entorno familiar. La calidad y disponibilidad de la información oportuna permite al paciente hacer la diferencia en la aceptación de una enfermedad difícil de llevar sin el acompañamiento requerido (Sánchez, y otros, 2019, pág. 1432).

En la mastoplastia de aumento, la complicación descrita con mayor frecuencia es la contractura capsular (CC), con una incidencia de $30 \%$, sin embargo, en las demás complicaciones (hematoma, seroma, infección y cicatriz inestética) es menor al $6 \%$. La CC es la complicación más común y frustrante en mujeres que se han sometido a cirugía de aumento mamario. Puede desarrollarse lenta o rápidamente. Se suele observar entre los 4-8 meses después de la cirugía, pero el riesgo de su aparición se extiende hasta varios años después (Figueroa, Trujillo, Varela, \& Carbón, 2020, pág. 3). Se han descrito técnicas variadas para controlar el dolor postoperatorio en este grupo de pacientes: infusiones continuas de anestésicos locales a través de catéteres, irrigación de anestésicos locales, como la bupivacaína, o de antiinfamatorios no esteroideos, como el ketorolaco, en la cavidad mamaria, analgesia por vía oral, analgesia de incisión, infiltración simple o continua de anestésicos locales, como la bupivacaína y la ropivacaína, administración de corticosteroides en el intraoperatorio, bloqueo paravertebral intraoperatorio y analgesia peridural simple o continua. Las técnicas que incluyen el uso por infiltración o en irrigación de los anestésicos locales, principalmente de lidocaína, bupivacaína o ropivacaína, se están utilizando ampliamente como parte de la analgesia multimodal en diferentes tipos de cirugías, con una efectividad notable y sin reportes de efectos adversos relacionados con los anestésicos locales (Jiménez Haad \& Chaves Vega, 2008).

Con los nuevos conceptos, enfoques, criterios de la cirugía estética y reconstructiva, surge la necesidad de la intervención del fisioterapeuta en la rehabilitación de los pacientes que han sido tratados en este campo de cirugías, ya que se ha detectado la necesidad de incorporar a profesionales de diferentes disciplinas del área de la salud en los procesos de intervención y rehabilitación oncológica, aunque actualmente es un campo poco explorado y pobremente manejado (Aguinaga Pinto, 2015). 


\section{Metodología}

La metodología de la investigación, es una revisión bibliográfica, que se apoyó en medios electrónicos disponibles en la web. Artículos científicos., páginas web, libros, fueron punto de partida importante para la extracción de la información utilizada para la construcción de este trabajo, en donde se reflejan aspectos importantes, sobre el tema de estudio. La idea fundamental es que sea punto de partida para futuras investigaciones.

\section{Resultados}

A la hora de afrontar una cirugía, el papel enfermero es fundamental para la preparación tanto del procedimiento anestésico, como del quirúrgico, haciendo acopio del instrumental, aparataje y materiales necesarios para la intervención quirúrgica. Enfermería acompaña y cuida de la paciente en las etapas preoperatoria inmediata, intraoperatoria y postoperatoria (López Madurga, 2020).

La enfermera de anestesia prepara la medicación necesaria para la inducción anestésica en colaboración con el anestesiólogo. Para una anestesia general será necesario:

- Premedicación: midazolam

- Inductor anestésico: Bromuro de rocuronio o cisatracurio

- Analgesia: fentanilo

- Tubo endotraqueal de tamaño adecuado a la paciente $(7-7,5)$

- Laringoscopio, Glidescope, Airtrack, fibroscopio

- Venda de gasa o esparadrapo para fijar tubo endotraqueal

- Lágrimas artificiales y protectores oculares

- Efedrina

- Atropina (López Madurga, 2020).

Previamente se habrá realizado la revisión de la torre de anestesia, sistemas de moni- torización, medicación ordinaria y extraordinaria intravenosa del carro de anestesia y del material necesario: equipos de gotero, bombas de perfusión y material fungible. La enfermera de anestesia canalizará una vía venosa y verificará la ausencia de prótesis, objetos de metal y esmalte de uñas y monitorizará a la paciente hemodinámicamente (T.A., electrocardiograma y pulsioximetría) (López Madurga, 2020).

Preparación del instrumental, material fungible y aparataje:

La preparación la realizan la enfermera instrumentista y circulante conjuntamente.

- Instrumental: Caja de mama con el siguiente instrumental

- Mosquitos

- Pinzas de Kocher

- Allis

- Portaagujas de distintos tamaños

- Mangos de bisturí n³ y 4

- Tijeras de Mayo y Metzenbaum

- Pinzas de Adson con dientes, sin dientes

- Pinzas de Gillies

- Pinzas de hemostasia

- Separadores: Senn-Miller, Roux y Tebbetts

- Areolotomo con medidas de 38 a 50 mm (si el abordaje es periareolar o hay que hacer algún retoque en areolas)

- Material fungible:

- Campo quirúrgico universal

- Mangos de luz de lámparas quirúrgicas

- Compresas, gasas

- Batas/guantes estériles

- Marcadores dérmicos

- Bisturí eléctrico (pieza de mano) y terminal largo de bisturí o prolongador de bisturí eléctrico

- Hojas de bisturí del no 15 y 20

- Azul de metileno, jeringa de $5 \mathrm{ml}$, aguja de carga y aguja intramuscular para tatuar puntos del marcaje dérmico y que no se borre durante la intervención

- Goma de aspiración y terminal mediano 
de yankauer

- Drenajes calibre 10, con sus receptales

- Apósitos

- Vendaje o sujetador

- Suturas:

- Sutura quirúrgica trenzada incolora reabsorbible de $3 / 0$ y 2/0

- Seda 2/0 triangular para fijar el drenaje

- Monofilamento incoloro reabsorbible de 4/0 con aguja triangular

- Tiras de aproximación

- Aparataje: consola de bisturí eléctrico y sistema de aspiración, comprobado su funcionamiento previamente a la entrada de la paciente en quirófano (López Madurga, 2020).

Una vez dentro de la sala de intervenciones, la instrumentista hará el lavado quirúrgico (OMS recomienda povidona iodada o clorhexidina jabonosa para el lavado quirúrgico o bien, lavado de manos con jabón y posteriormente aplicación de solución hidroalcohólica durante al menos 90 segundos) y preparará el instrumental y todo el material estéril necesario para la intervención (López Madurga, 2020).

\section{Tipos de heridas}

Herida limpia: Es una herida no infectada, en la cual no hay inflamación significativa y los tractos respiratorios, digestivo, genital o urinario no se manipulan. Adicionalmente incluye una técnica de disección roma y la no ruptura de las normas de asepsia y antisepsia (Flórez Velasquez, 2018).

Herida Limpia- contaminada: Implica manipulación y entrada en el tracto respiratorio, digestivo (excluyendo intestino), genital o urinario no infectado; y no presenta ruptura mayor de la técnica estéril (Flórez Velasquez, 2018).

Es así como la mamoplastia de aumento debe considerarse una herida limpia - contaminada, con un riesgo aumentado para infección del sitio operatorio por la presen- cia de implante y se recomienda el uso de cefazolina $1 \mathrm{~g}$ IV en los 60 minutos previos a la intervención quirúrgica; en pacientes alérgicos a B-lactámicos se recomienda el uso de clindamicina $900 \mathrm{mg}$ IV en reemplazo a la cefazolina. Si bien es infrecuente que suceda, cuando el tiempo quirúrgico es mayor a 4 horas, se debe repetir la dosis del antibiótico profiláctico y no se recomienda profilaxis adicional posoperatoria (Flórez Velasquez, 2018).

\section{Tipos de infecciones}

Infecciones agudas: Son las infecciones posoperatorias más comunes, usualmente se dan entre la primera y la sexta semana después de la cirugía, aunque, algunos autores la definen hasta los 3 primeros meses posoperatorios. Otros autores encasillan el período entre la sexta semana y los 3 meses como infecciones subagudas, sin embargo, dicha división no es útil en la práctica clínica, por lo que no se recomienda realizarla. El espectro de los microorganismos más frecuentemente encontrados incluye en casi su totalidad a gram positivos, el S. aureus Meticilino Sensible (SAMS) y el S. epidermidis son los microorganismos que se encuentran de manera más consistente en la literatura, con incidencias aproximadas al $40 \%$ para cada uno de ellos (Flórez Velasquez, 2018).

Infecciones tardías: Se presentan aproximadamente desde los 3 meses posoperatorio hasta años después. Son poco comunes y se manifiestan típicamente como dolor vago en las mamas con o sin cambios inflamatorios en la piel, empero, pueden progresar también a signos y síntomas sistémicos. Generalmente se han asociado indistintamente con microorganismos gram positivos y gram negativos como el Streptococcus pyogenes, Clostridium perfringens y Klebsiella pneumoniae (Flórez Velasquez, 2018). TREJOS, BELTRÁN, PINZÓN, MARÍN, \& SUAREZ, (2018) citan a Araco y cols (2007) cuando estos menciona otras complicacio-

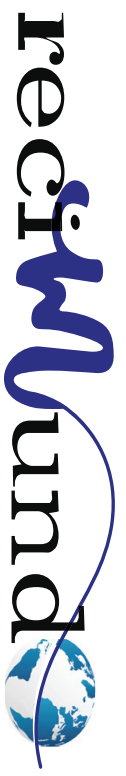


nes presentes luego de una mamoplastia, que fueron encontradas luego de realizarse un estudio a 3.002 mujeres, y los resultados fueron los siguientes:

Hematoma: esta complicación estuvo presente en el $1.5 \%$ de los pacientes. Se evidenció que la mayoría de pacientes que presentaron esta complicación tenían implantes en localización submuscular usando tanto el abordaje clásico como el de plano dual. El análisis de regresión logística multivariado confirmó que los únicos factores de riesgo que incrementan la formación de hematoma son el plano de colocación de la prótesis y la disección manual del bolsillo. Infección: La infección se encontró en 1.1\% de los pacientes. Se evidenció una ligera prevalencia en la localización subglandular $(54.5 \%)$ versus submuscular/plano dual (45.4\%).

Asimetría mamaria: esta complicación estuvo presente en $0.8 \%$ de los pacientes. La mayoría tienen prótesis de localización submuscular colocadas mediante abordaje clásico o de plano dual. Adicionalmente se observó una prevalencia en pacientes con implantes con volumen mayor a $280 \mathrm{cc}$.

Ondulación (rippling): esta estuvo presente en $0.7 \%$ de los pacientes. La mayoría tenían implantes de localización subglandular (81\%). El 66\% tenían disección manual del bolsillo y colocación de dren posoperatorio. Contractura capsular: esta fue la complicación observada con menor frecuencia, presentándose solo en $0.5 \%$ de los casos. La mayoría de pacientes con contractura capsular tenían implantes de localización subglandular. El $64.3 \%$ tenían implantes con volúmenes altos de 325 a 350cc y al $92.8 \%$ se le realizó disección manual del bolsillo y colocación de dren (pág. 70).

\section{Cuidados post operatorios}

- Finalizada la cirugía de la mama con anestesia general es recomendable permanecer hospitalizada por 24 horas, con el cabecero levemente inclinado para impedir que la paciente dormida se tumbe de lado. Esta posición mejora el drenaje de los tejidos mamarios y reduce la inflamación. La paciente no debe realizar movimientos bruscos con los brazos, aunque no sienta dolor. El no guardar reposo así la paciente no tenga molestia alguna, puede desencadenar complicaciones como rotación de la prótesis en caso de mamoplastias de aumento, o hemorragias en cualquier tipo de intervención, aumento el riesgo de cirugías secundarias. En caso de una reconstrucción con colgajos libres o pediculados, se debe evitar comprimir toda la zona intervenida para no colapsar el pedículo que vasculariza el colgajo (Sánchez, y otros, 2019).

- Dada de alta hospitalaria la paciente debe cumplir exhaustivamente las pautas de medicación que le mande su cirujano, esencialmente analgésicos, antiinflamatorios y en ocasiones relajantes musculares y heparina. No debe mojar las incisiones, preservándolas limpias y pinceladas con algún antiséptico de manera frecuente. No fume al menos, hasta que no se hayan retirado las suturas, siendo ideal el cese completo del hábito tabáquico. En caso de necesitar alguna prenda de presoterapia como sujetador ortopédico o fajas, debe usarlos las 24 horas del día durante el primer mes para prevenir el desarrollo de seromas y para fijar las cápsulas en caso de cirugías que incluyan prótesis. Sólo deberá quitarse estas prendas para la higiene diaria (Sánchez, y otros, 2019).

La operación del aumento de pecho se llevará a cabo bajo los efectos de la anestesia general y tendrá una duración aproximada de entre 1 y 2 horas. Una vez la paciente despierte, esta será mantenida en observación por el personal del hospital y podrá retornar a casa tras una o dos noches (https:// www.quironsalud.es, 2019). 
Es normal que durante la primera semana se padezcan algunos dolores y se note cierto nivel de inflamación en la zona. Todo esto será oportunamente atendido por los profesionales sanitarios a cargo del proceso, quienes recetarán los medicamentos necesarios para reducir los síntomas y hacer más llevadera la recuperación (https://www. quironsalud.es, 2019).

Si bien en estos primeros días será necesario guardar cierto reposo, a partir de la segunda semana ya se podrán retomar las actividades cotidianas con normalidad. De todas maneras es importante no exigir demasiado al cuerpo, además de utilizar un sujetador especial que ayude a sanar los senos y a mantener los resultados obtenidos (https://www. quironsalud.es, 2019).

\section{Conclusiones}

La mamoplastia es una operación que se realiza en la mayoría de los casos de manera estética, ya que sencillamente es un aumento de los senos, sin embargo también puede realizarse para la reducción de los mismos (mamoplastia de reducción) y como consecuencias de mastectomías derivadas del cáncer. Como todo procedimiento quirúrgico que se desarrolla en un quirófano, este no está exento de generar complicaciones en las pacientes, sin embargo la incidencia de las mismas son muy bajas $(4,6 \%)$.

De presentarse incidencias los hematomas y seromas son los más comunes. Sin embargo los cuidados quirúrgicos que se deben preservar luego de la operación, están asociados al cuido del mismo paciente y su entorno, ya que luego de realizarse e procedimiento de mamoplastia, la paciente no dura más de 24 horas hospitalizada, esto quiere decir, que la mayoría de las complicaciones se generan fuera del centro de salud. Esto debido en muchos casos por que no se siguen los protocolos de cuidado que recomienda el cirujano.
El protocolo de tratamiento que se manda a casa es analgésicos y antiinflamatorios. En algunos casos hay hematomas que salen por la manipulación de la piel al insertar las prótesis pero van desapareciendo a las semanas sin mayores complicaciones.

\section{Bibliografía}

Aguinaga Pinto, Y. R. (2015). Demostración de la eficacia de un correcto abordaje kinefisioterapéutico en el post quirúrgico de la mamoplastia reconstructiva en pacientes con cáncer de mama.

Figueroa, D. K., Trujillo, A., Varela, C., \& Carbón, A. (2020). Caracterización de las pacientes con contractura capsular operadas de mastoplastia de aumento con implante. Acta Médica, 21(3).

Flórez Velasquez, S. A. (2018). Manejo antibiótico en mamoplastia de aumento. Departamento de Cirugía.

https://www.quironsalud.es. (06 de 09 de 2019). Obtenido de https://www.quironsalud.es/blogs/es/ tendencia-estetica/postoperatorio-aumento-pecho

Jiménez Haad, R., \& Chaves Vega, A. (2008). Irrigación de anestésicos locales y dolor postoperatorio en pacientes sometidas a mamoplastia de aumento. Colombian Journal of Anestesiology, 36(3), 167-172.

López Madurga, S. (12 de 08 de 2020). Obtenido de https://www.revista-portalesmedicos.com/ revista-medica/funciones-y-cuidados-perioperatorios-de-enfermeria-en-la-cirugia-de-aumento-de-mamas/

Prieto, I. R., Jerez, L., Pineda, L., \& Robayo, F. (2014). Mamoplastia De Aumento: Complicaciones Y Manejo Postquirúrgico Desde Fisioterapia. CATUSSABA-ISSN 2237-3608, 3(2), 37-49.

Sánchez, E. J., Mieles, J., Alcívar, A., Robles, M., Imbaquingo, F., \& Loor, A. (2019). Prevención y cuidados post operatorios de las mujeres sometidas a cirugía de cáncer de mama. RECIAMUC, 3(3), 1429-1443. doi:https://doi.org/10.26820/reciamuc/3.(3).julio.2019.1429-1443

TREJOS, S. O., BELTRÁN, E., PINZÓN, J., MARÍN, J., \& SUAREZ, R. (2018). Disminución del riesgo en cirugía plástica. Recomendaciones. Parte II: contorno corporal. Revista Colombiana de Cirugía Plástica y Reconstructiva, 24(1), 64-74. 
DIA, L. G.
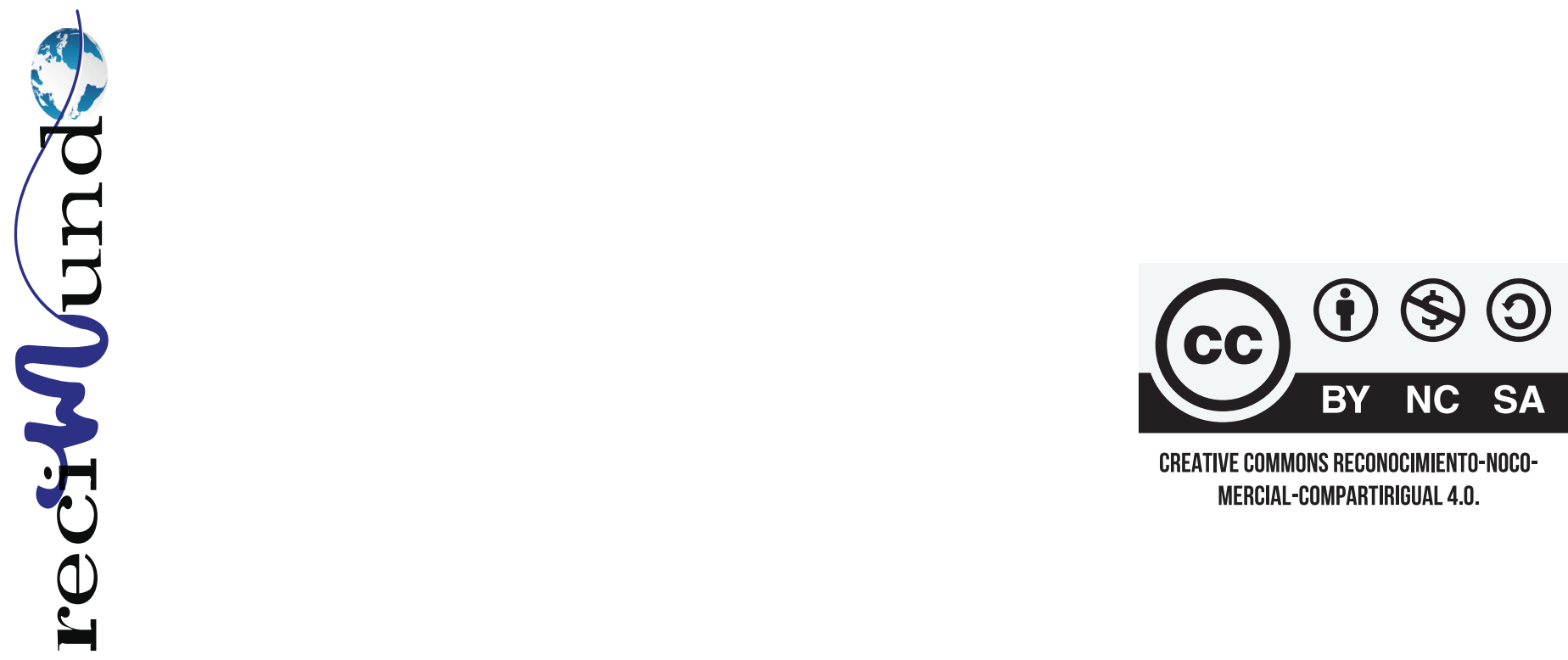

CREATIVE COMMONS RECONOCIMIENTO-NOCOMERCIAL-COMPARTIRIGUAL 4.0.

\section{CITAR ESTE ARTICULO:}

Zambrano Zambrano, F. C., Campozano Burgos, M. A., Cedeño Flores, F. P., \& Chávez Heredia, L. G. (2021). Cuidados quirúrgicos a pacientes sometidos a una mamoplastia. RECIMUNDO, 4(4), 532-538. https://doi.org/10.26820/recimundo/4.(4).octubre.2020.532-538 